\title{
Tumor Interstitial Fluid Pressure Measurement
}

National Cancer Institute

\section{Source}

National Cancer Institute. Tumor Interstitial Fluid Pressure Measurement. NCI

Thesaurus. Code C116703.

The act of quantifying the fluid pressure inside a tumor. 